\title{
A Method for Measuring the Accuracy of the S Part Side Wall Surfaces Programming
}

\author{
Yu Peng ,Chao Ruan
}

Chengdu Aircraft Industry (Group) Co., Ltd,Chengdu,610091,China

vencent@126.com

Keywords: S Part, Vericut , Simulation, Programming Accuracy

Abstract: S part side wall surfaces programming is always a technical problem for the Manufacturing enterprise users, this paper proposes a method for measuring the accuracy of the $S$ part side wall surfaces programing in the cutting process before, and can form a precision inspection report, provide the industry with a valid measuring method for $S$ part side wall surfaces programming accuracy.

\section{Introduction}

$\mathrm{S}$ part is a dynamic precision test piece for multi axis machine tool proposed by Chengdu Aircraft Industry (Group) Co., Ltd., its core content is through the machine test cut of S part in the side wall surface, multi axis precision and detection as the main object to indirect inspection machine and system. The surface of the side wall of the part is a non developable straight surface formed on the basis of two interlaced spline curves above and below, as shown in Figure 1. The contour of the formed surface is less than equal to $0.12 \mathrm{MM}$. Due to the complex changes in the process of the surface of angle, so the $\mathrm{S}$ part is used to test the machine debugging after the completion of the dynamic multi axis machining accuracy, in order to evaluate the reliability of machine tool products before processing formally.
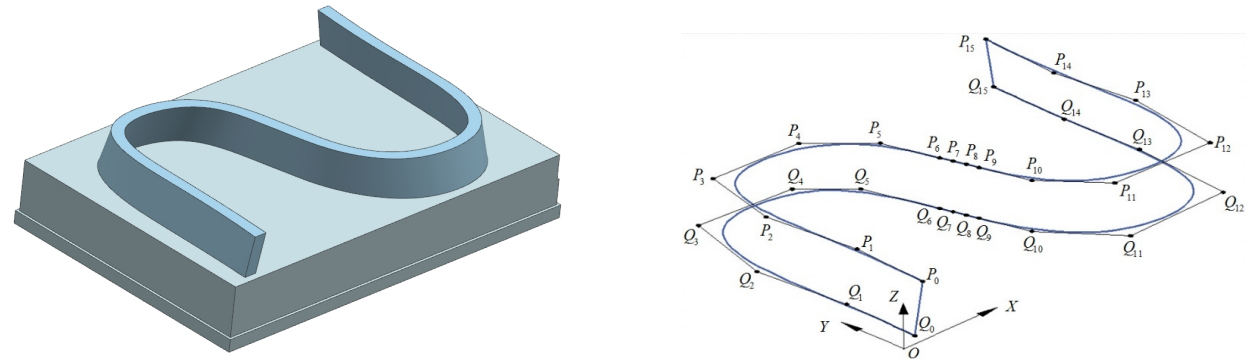

Figure 1 flying S part and its side wall surfaces composition

With the popularization and application of multi axis CNC machine tools in recent years, more and more manufacturing enterprises and machine manufacturers pay more attention to the cutting quality and cutting performance of their multi axis machine tools in the process of S part. It can be no exaggeration to say that the machining quality of $\mathrm{S}$ part, especially the s part side wall surface programing, has become a key technical index to weigh a manufacturing enterprise and machine tool manufacturer.

In view of this, the author takes the experience in the international standard setting process of $S$ part into account, and puts forward a method for testing the accuracy of $S$ part side wall surfaces programming. It can check the multi axis machining procedure of S part before machining. 


\section{Drawback of the current method}

The machined contour error of the $\mathrm{S}$ part side wall surfaces is $\pm 0.06 \mathrm{MM}$, which requires high accuracy control for $\mathrm{S}$ part modeling, programming and machining. From the general cognition of the industry, the accuracy of S part should be controlled within $0.01 \mathrm{MM}$, otherwise it will not be able to effectively guarantee the accuracy of the final S part surface processing, which challenges the accuracy and detection method of S part.

According to the common programming software and programming methods, the programming error of the multi axis side milling of the side wall surfaces of the $\mathrm{S}$ part is more than $0.015 \mathrm{MM}$ in general. The main reasons are as follows:

1)The $S$ part surface side milling programming process, due to the level of user programming programming is uneven, parameter setting is not reasonable, resulting in side milling path accuracy is not up to the requirements of the processing, but the CAM system has not been cut or excess warning; or only over cut and left that simple, unable to accurately locate the after cutting, the excess part.

2)The surface of the CAM software of multi axis side milling programming is generally general method, not high precision programming of $\mathrm{S}$ part requires complete response, accurate simulation and analysis of its own function to get the simulation program of multi axis side milling tool path.

3)The general user simulation software - free surface multi axis side milling of $S \mathrm{~S}$ looks at the integrity of the free surface, the free surface precision of simulation can not be formed accurate analysis.

\section{Proposed method based on Vericut}

The main steps of the method proposed in this paper are as follows:

1) Simulate and Get the result model of the side wall surfaces finishing of $S$ part based on Vericut software simulation

2) Create high density measurement points based on the side wall surfaces of the S part, and get the APT file for measurement.

3) Custom development simulation test program

4) Simulate and form a test report

Its typical processing flow chart is as follows:

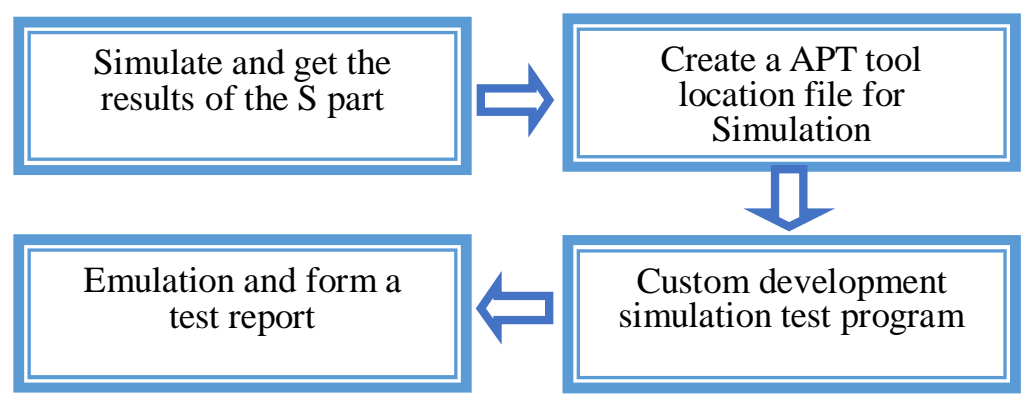

Figure 2 Work flow of the proposed method

\section{Typical example description and verification}

Based on the CATIA software of $\mathrm{S}$ part side wall surfaces finishing program as an example to illustrate how to program precision detection, in order to reflect the general, the multi axis side wall 
of the most commonly used software programming method for S part side wall finishing program, whether to verify the method proposed in this paper can detection of the programming method of weave programming precision to meet the requirements. The detected points are from the theoretical data points on the same S part model surface, and the tool used is cylindrical milling cutter with a diameter of 20 and a working length of 50MM.

\section{Step 1 :Get the model of the precision machining result of $S$ part}

With the help of the general sidewall multi axis programming method of CATIA software, it is easy to get the cutter location file of the $S$ part. Then, the simulation environment is built in the simulation software Vericut, and the simulation result model of the $\mathrm{S}$ part is simulated, as shown in Figure 3.

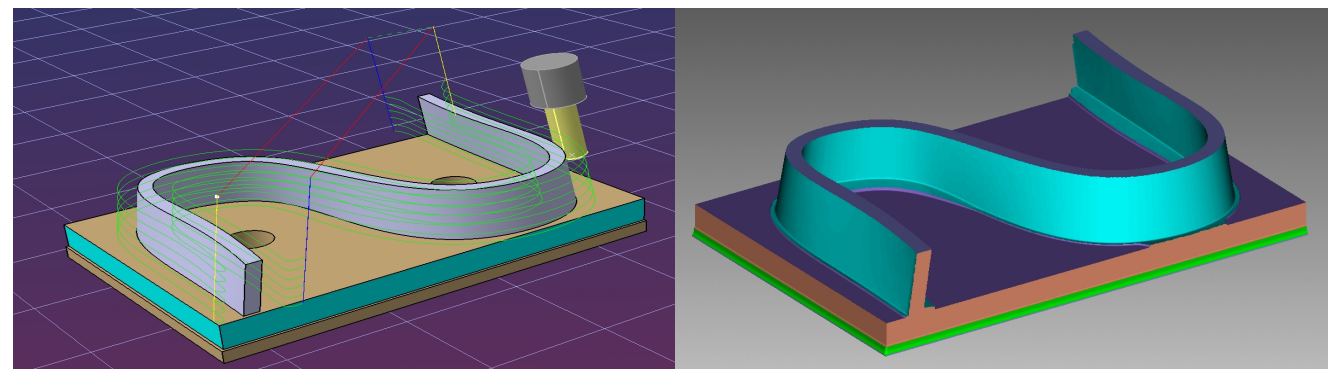

Figure 3 simulation effect diagram for example parts

\section{Step2 :Create the measurement point on the surface and generate the points file}

According to the measurement of the point rule of the side wall surfaces of the $\mathrm{S}$ part, some measurement points can be generated on the surface in the CATIA environment, as shown in Figure 4. The number of points can be chosen according to the precision requirement of the detection. In general, the higher the requirement of programming precision, the more measurement points are needed. After the point position is built, the XYZ coordinates of each point and the normal vector IJK of the corresponding surface position are shown, as shown in Figure 4 left

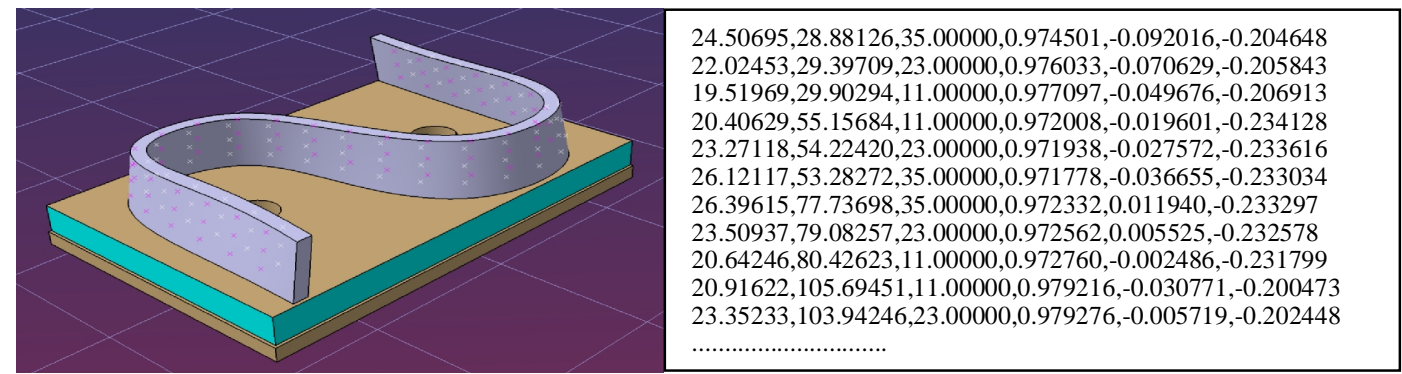

Figure 4 optimal parameter information of a tool for a target machine tool

\section{Step 3:Custom development simulation test program}

Because the output points file is the theoretical point of side wall on the surface, the actual measuring head simulation measurement plane, also need to consider the safety of point measurement in the process of contact and contact direction of safety distance, probe the practical working principle as shown in Figure 5 are left, two times the development of VERICUT software based on combining the measuring module customization development simulation test program generation probe detection control simulation subroutine for the TM, as shown in Figure 5 is shown on the right. 


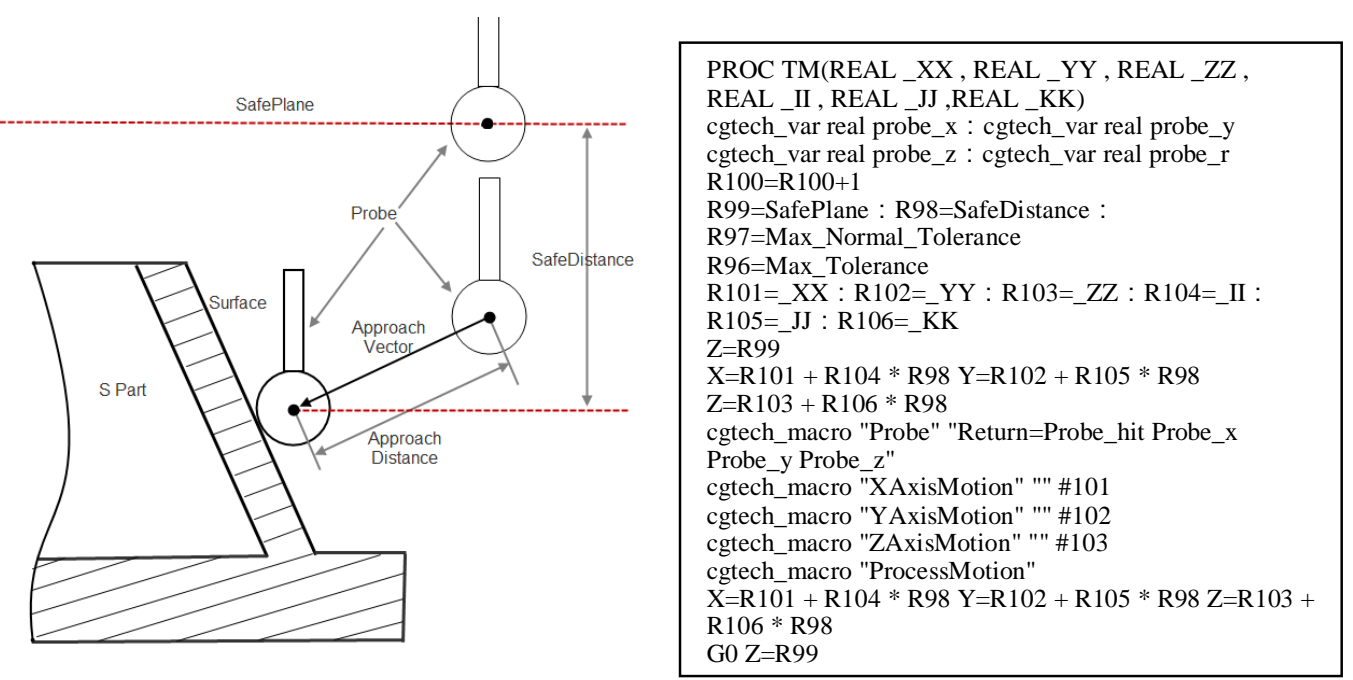

Figure 5 Comparison of numerical control programs before and after optimization

\section{Step 4: Simulate and form a test report}

Based on the above measurement program and the measurement point position, the measurement code for each point is generated, as shown in Figure 6. In the Vericut simulation and implementation of the automatic detection of each point and the output of the detection report, see Figure 7:

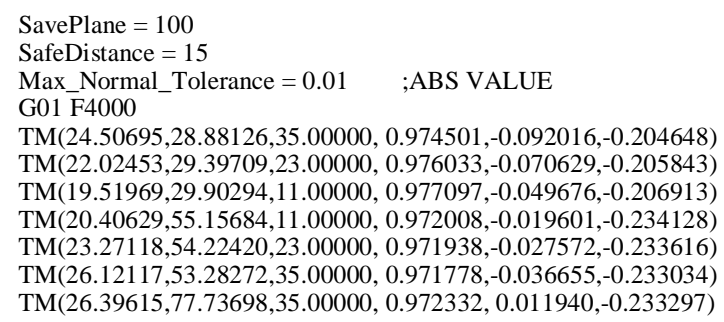

TM(24.50695,28.88126,35.00000, 0.974501,-0.092016,-0.204648) TM(22.02453,29.39709,23.00000, 0.976033,-0.070629,-0.205843) TM(19.51969,29.90294,11.00000, 0.977097,-0.049676,-0.206913) TM(20.40629,55.15684,11.00000, 0.972008,-0.019601,-0.234128) $\operatorname{TM}(23.27118,54.22420,23.00000,0.971938,-0.027572,-0.233616)$ $\operatorname{TM}(26.12117,53.28272,35.00000,0.971778,-0.036655,-0.233034)$ $\mathrm{TM}(26.39615,77.73698,35.00000,0.972332,0.011940,-0.233297)$

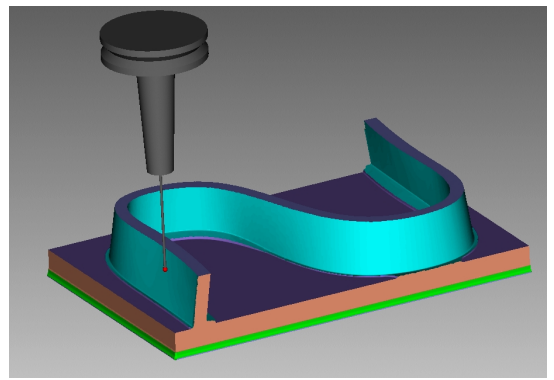

Figure 6 Measurement code and simulation based on Vericut

\begin{tabular}{|c|c|c|c|c|c|c|c|c|}
\hline \multicolumn{9}{|c|}{ 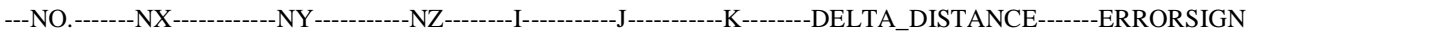 } \\
\hline *POINT:1 & 24.50695 & 28.88126 & 35 & -0.974501 & 0.092016 & 0.204648 & -0.0003 & \multirow{5}{*}{ OverTolerance } \\
\hline *POINT:2 & 22.02453 & 29.39709 & 23 & -0.976033 & 0.070629 & 0.205843 & 0.0042 & \\
\hline *POINT:3 & 19.51969 & 29.90294 & 11 & -0.977097 & 0.049676 & 0.206913 & 0.012 & \\
\hline *POINT:4 & 20.40629 & 55.15684 & 11 & -0.972008 & 0.019601 & 0.234128 & 0.0009 & \\
\hline *POINT:5 & 23.27118 & 54.2242 & 23 & -0.971938 & 0.027572 & 0.233616 & 0.0003 & \\
\hline *POINT:6 & 26.12117 & 53.28272 & 35 & -0.971778 & 0.036655 & 0.233034 & -0.0007 & \multirow{8}{*}{ OverTolerance } \\
\hline *POINT:7 & 26.39615 & 77.73698 & 35 & -0.972332 & -0.01194 & 0.233297 & -0.0004 & \\
\hline *POINT:8 & 23.50937 & 79.08257 & 23 & -0.972562 & -0.005525 & 0.232578 & 0.0004 & \\
\hline *POINT:9 & 20.64246 & 80.42623 & 11 & -0.97276 & 0.002486 & 0.231799 & 0.0024 & \\
\hline *POINT:10 & 20.91622 & 105.69451 & 11 & -0.979216 & 0.030771 & 0.200473 & 0.015 & \\
\hline *POINT:11 & 23.35233 & 103.94246 & 23 & -0.979276 & 0.005719 & 0.202448 & 0.0056 & \\
\hline *POINT:12 & 25.85726 & 102.18911 & 35 & -0.978714 & -0.018553 & 0.204386 & 0.0008 & \\
\hline *POINT:13 & 26.50174 & 126.62483 & 35 & -0.982213 & 0.093939 & 0.162583 & 0.0033 & \\
\hline
\end{tabular}

Figure 7 The test report obtained by simulation

\section{Conclusion}

As can be seen from Figure 7, the general program of the general multi axis side milling programming method based on CATIA is still available, with the smallest error reaching the nanometer level, but there are still more than $0.01 \mathrm{~mm}$ measuring points. This proves that the accuracy of the programming method is effective, and it also reminds users to check the programming accuracy and prevent the programming error from being too large when programing 
the $\mathrm{S}$ part side wall surface, so as to provide effective guarantee for the $\mathrm{S}$ part's actual machine tool processing qualification.

In addition, the method proposed in this paper can not only quickly know whether the side milling program of S part is up to the requirements, but also can be extended to other high precision parts in the process of programming accuracy detection, which is an effective general programming accuracy detection method.

\section{Acknowledgement}

This work was partially supported by National Science and Technology Major Project of China (2014ZX04014-031).

\section{Reference}

[1] DING Jiexiong,TAN Yang,etc.Study on Processing and Displaying Contour Error at Tesing Specimen for Five-xis Machine Tool[J],Modular Machine Tool \& Automatic Manufacturing Technique,2012(10):39-43.

[2] PENG Yu,MOU Wenping.Optimization Strategy of NC Programming for S Part Based on NX[J],Intelligent Manufacturing,2013(9):81-83. 